\title{
Twelve years of experience with miglustat in the treatment of type 1 Gaucher disease: The Spanish ZAGAL project
}

\author{
Pilar Giraldo a,b,c,d,*, Marcio Andrade-Campos ${ }^{\text {a,b,c,d }}$, Pilar Alfonso ${ }^{\text {b,c }}$, Pilar Irun ${ }^{\text {b,e }}$, Koldo Atutxa ${ }^{\text {, }}$ \\ Antonio Acedo ${ }^{\mathrm{g}}$, Abelardo Barez ${ }^{\mathrm{h}}$, Margarita Blanes ${ }^{\mathrm{i}}$, Vicente Diaz-Morant ${ }^{\mathrm{j}}$, Ma Angeles Fernández-Galán ${ }^{\mathrm{k}}$, \\ Rafael Franco ${ }^{1}$, Cristina Gil-Cortes ${ }^{\mathrm{m}}$, Vicente Giner ${ }^{\mathrm{n}}$, Angela Ibañez ${ }^{\mathrm{o}}$, Paz Latre ${ }^{\mathrm{c}}$, Ines Loyola ${ }^{\mathrm{p}}$, Elisa Luño ${ }^{\mathrm{q}}$, \\ Roberto Hernández-Martin ${ }^{\mathrm{r}}$, Blanca Medrano-Engay ${ }^{\mathrm{d}}$, José Puerta ${ }^{\mathrm{s}}$, Inmaculada Roig ${ }^{\mathrm{t}}$, Javier de la Serna ${ }^{\mathrm{u}}$,

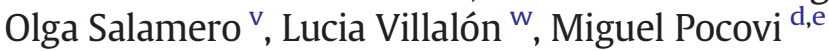

a Haematology, Miguel Servet University Hospital, Zaragoza, Spain

b CIBER de Enfermedades Raras (CIBERER), Spain

c Spanish Foundation for the Study and Therapy of Gaucher Disease (FEETEG), Spain

${ }^{\mathrm{d}}$ Institute of Health Research Aragon (IIS Aragón), Zaragoza, Spain

e Biochemistry and Molecular and Cellular Biology, Zaragoza University, Spain

${ }^{\mathrm{f}}$ Haematology, Galdakao Hospital, Usansolo, Spain

${ }^{g}$ Haematology, Vega Baja Hospital, Alicante, Spain

${ }^{\text {h }}$ Haematology, Ntra Sra de Sonsoles Hospital, Avila, Spain

i Haematology, Virgen de la Salud Hospital, Elda, Spain

j Internal Medicine, Ronda Hospital, Ronda, Spain

${ }^{\mathrm{k}}$ Virgen del Puerto Hospital, Plasencia, Spain

${ }^{1}$ Haematology, Punta Europa University Hospital, Cádiz, Spain

${ }^{\mathrm{m}}$ Haematology, San Vicente Hospital, Alicante, Spain

${ }^{\mathrm{n}}$ Internal Medicine, Virgen de Los Lirios Hospital, Alcoy, Spain

o Haematology, Complejo Hospitalario de Albacete, Spain

p Haematology, Complexo Hospitalario de Pontevedra, Spain

${ }^{q}$ Haematology, Central de Asturias University Hospital, Oviedo, Spain

${ }^{r}$ Haematology, Virgen de la Concha Hospital, Zamora, Spain

${ }^{s}$ Haematology, Virgen de las Nieves Hospital. Granada, Spain

${ }^{\mathrm{t}}$ Haematology, Parc Tauli Hospital, Sabadell, Spain

u Haematology, Doce de Octubre University Hospital, Madrid, Spain

Haematology, Vall d'Hebron University Hospital, Barcelona, Spain

${ }^{w}$ Haematology, Alcorcon Hospital, Madrid, Spain

\section{A R T I C L E I N F O}

\section{Article history:}

Submitted 21 August 2016

Revised 22 October 2016

Accepted 22 October 2016

Available online $\mathrm{xxxx}$

\section{Keywords:}

Gaucher disease type 1

Miglustat

Efficacy

Safety

Maintenance

\begin{abstract}
A B S T R A C T
We report data from a prospective, observational study (ZAGAL) evaluating miglustat $100 \mathrm{mg}$ three times daily orally. in treatment-naïve patients and patients with type 1 Gaucher Disease (GD1) switched from previous enzyme replacement therapy (ERT). Clinical evolution, changes in organ size, blood counts, disease biomarkers, bone marrow infiltration (S-MRI), bone mineral density by broadband ultrasound densitometry (BMD), safety and tolerability annual reports were analysed. Between May 2004 and April 2016, 63 patients received miglustat therapy; 20 (32\%) untreated and 43 (68\%) switched. At the time of this report 39 patients (14 [36\%] treatment-naïve; 25 [64\%] switch) remain on miglustat. With over 12-year follow-up, hematologic counts, liver and spleen volumes remained stable. In total, $80 \%$ of patients achieved current GD1 therapeutic goals. Plasma chitotriosidase activity and CCL-18/PARC concentration showed a trend towards a slight increase. Reductions on S-MRI $(\mathrm{p}=0.042)$ with an increase in BMD $(\mathrm{p}<0.01)$ were registered. Gastrointestinal disturbances were reported in $25 / 63$ (40\%), causing miglustat suspension in 11/63 (17.5\%) cases. Thirty-eight patients (60\%) experienced a fine hand tremor and two a reversible peripheral neuropathy. Overall, miglustat was effective as a long-
\end{abstract}

Abbreviations: BUA, broadband ultrasound attenuation; BMD, bone mineral density; CCL18/PARC, chemokine (C-C motif) ligand 18/pulmonary and activation-regulated chemokine;

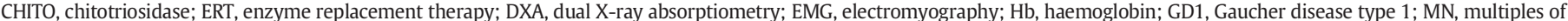
normal; SD, standard deviation; SE, standard error; S-MRI, Spanish semi-quantitative MRI; SOS, speed of sound.

* Corresponding author at: Translational Research Unit, Miguel Servet University Hospital/IACS, P Isabel La Católica 1-3, 50009 Zaragoza, Spain.

E-mail address: giraldocastellano@gmail.com (P. Giraldo). 
term therapy in mild to moderate naïve and ERT stabilized patients. No unexpected safety signals were identified during 12 -years follow-up.

\section{Background}

Type 1 Gaucher disease (GD1) (OMIM 230800) is an autosomal-recessive inborn disorder of lysosomal metabolism characterized by the accumulation of glucocerebroside in different organs and systems as a consequence of the deficient activity of the enzyme, glucocerebrosidase (acid $\beta$-glucosidase). GD1 is a multisystemic disease associated with a considerable degree of heterogeneity in terms of symptoms type and severity. It is also one of the most common lysosomal storage disorders, and the first for which multiple approved therapies were available [1,2].

Enzyme replacement therapy (ERT) has been used widely to treat GD1 since 1990 and, in general, studies have demonstrated good efficacy and favourable safety/tolerability [3,4,5]. The achievement and maintenance of GD1 therapeutic goals is considered a useful way to monitor clinical efficacy, and good rates of goal achievement have been reported in a number of clinical studies assessing ERT [6,7]. However, some GD1 patients on long-term ERT can develop a plateau in their response to ERT in terms of haematological and visceral disease parameters. The objective of continued treatment in such patients is to maintain symptom control and ensure adequate quality of life using the most cost-effective long-term treatment $[6,7,8,9,10]$.

Miglustat (Zavesca ${ }^{\circledR}$; Actelion Pharmaceuticals) is an orally active iminosugar that reversibly inhibits UDP-glucosylceramide synthase - the enzyme that catalyses the rate-limiting stage in the glycosphingolipid biosynthesis pathway [11]. Miglustat was approved in Europe in March 2003 and in the USA in 2004 for use as a substrate reduction therapy (SRT) in adult patients with mild-to-moderate GD1 for whom ERT is either unsuitable or is not a therapeutic option $[12,13,14]$.

There are a number of published data sets on clinical experience with miglustat in GD1, the majority of which report findings from clinical trials, multicentre retrospective cohorts and case series $[15,16,17$, $18,19]$. In Spain the prospective, observational ZAGAL study was initiated by the Spanish Gaucher Disease Foundation after miglustat was approved by the European Medicines Agency in 2004. We have previously reported ZAGAL study data regarding the effects of both shortterm (6-month) and long-term (5-year) miglustat therapy during everyday clinical use in treatment-naïve patients and those previously treated with ERT $[15,16]$. Here, we report the long-term efficacy, safety and tolerability of miglustat in ZAGAL study patients with mild-to-moderate GD1 treated in clinical practice settings during a follow-up period of 12 years.

\section{Methods}

\subsection{Study design}

The ZAGAL study is a prospective observational study that was initiated to establish a set of recommendations for the collection of efficacy, safety and quality-of-life data in a structured longitudinal manner, and to coordinate the use of miglustat for the treatment of GD1 in real-life settings. All consecutive patients with confirmed GD1 who fulfilled criteria to receive miglustat therapy are included. The study was designed in accordance with recommendations from the European Working Group on GD Advisory Council [20], and was subsequently approved by the Ethics Committee for Clinical Investigation of Aragon (CEICA) and was conducted in accordance with the Declaration of Helsinki of 1975, as revised in 2008 .

\subsection{Study treatment}

Miglustat therapy was administered according to guidelines devised by the Spanish Foundation for the Study and Therapy of Gaucher Disease (FEETEG) in order to optimize and standardize miglustat use. All treated patients received miglustat $100 \mathrm{mg}$ three times daily orally, with dietary recommendations to exclude carbohydrates during the first weeks of starting therapy.

\subsection{Efficacy and safety assessments}

Specific assessment methods have been described in detail in previous publications $[15,16]$. Effects on core GD disease parameters were considered in relation to established therapeutic goals for anaemia, thrombocytopenia, hepatomegaly and splenomegaly according to reports published by Pastores et al. [6,7]. Specific assessment methods have been described in detail in previous publications $[15,16]$.

Briefly, organ volumes were evaluated annually at local sites, and were corroborated in a subset of patients using magnetic resonance imaging (MRI). Bone marrow infiltration was evaluated every 12 months based on the published S-MRI scoring protocol for Gaucher cell infiltration patterns in the spine, pelvis and femur [21]. Bone mineral density (BMD) was evaluated using ultrasound measurements in both heels (calcaneus bone) using a Cuba Clinical Bone Densitometer (Norland Medical Systems). Broadband ultrasound attenuation (BUA), speed of sound (SOS), and estimated BMD were all determined at baseline and after 2 and 4 years of therapy. Plasma chitotriosidase (CHT) activity and CLL18/PARC concentration were analysed in the FEETEG laboratory using a 4-methylumbelliferyl-b-D-N, $\mathrm{N}^{\prime}, \mathrm{N}^{\prime \prime}$ triacetylchitotrioside substrate and enzyme-linked immunosorbent assay, respectively. Safety and tolerability assessments were based on patient- and treating-physician reports.

\subsection{Data analysis}

Descriptive statistics for continuous variables, sample size (n), mean and its standard error (SE), standard deviation (SD), median, and range were calculated for haemoglobin ( $\mathrm{Hb})$ concentration, platelet count, spleen volume, liver volume, and changes in biomarker activity. Spleen and liver volumes were calculated as multiples of normal (MN; where normal spleen volume is $2 \mathrm{~mL} / \mathrm{kg}$ of body weight and normal liver volume is $25 \mathrm{~mL} / \mathrm{kg}$ of body weight). Patient numbers and percentages were calculated for categorical variables based on patients with available data per time point.

Bone parameters were analysed by study cohort quartiles. KaplanMeier analysis estimate of the probability of drug discontinuation or death. Changes between pre- and post-therapy S-MRI scores were assessed using the paired $t$-test. For all statistical tests, differences were considered significant if $p<0.05$.

\section{Results}

\subsection{Patients and disposition}

To date, a total of 351 GD1 patients have been diagnosed in Spain [22]. Between 2004 and May 2016, 63 patients from 43 hospitals were treated with miglustat, among whom 20 (32\%) received miglustat as first-line therapy and 43 (68\%) switched from previous ERT (mean [range] ERT duration 6.9 [1.2-13.5] years). 
Baseline patient and disease characteristics for all treated patients, grouped according to treatment path (i.e., treatment naïve or switched), are summarized in Table 1. Overall, the mean (range) age at the time of GD1 diagnosis was 34.8 (2-79) years and the mean (range) age at initiation of miglustat therapy was 43.7 (18-79) years.

Of 63 patients exposed to miglustat, a total of 24 discontinued treatment during the study observation period: 11 due to uncontrolled gastrointestinal disturbances, six due to an increase in bone infiltration (two of whom had a bone crisis), one patient due to weight loss $>10 \%$, one due to persistent headache (during the early stages of treatment), and two due to planned pregnancy.

Five patients died during the study observation period due to reasons that were not considered related to treatment, including two cases of cancer in treatment-naïve patients (melanoma after 2 years and endometrial adenocarcinoma after 3 years of follow up), and one case each of myocardial infarction, liver failure and progressive general deterioration in switch patients.

Currently, 39 (62\%) patients continue to receive miglustat therapy (mean [range] age 44.2 [20-68] years; $41.0 \%$ female), among whom $14(36 \%)$ were treatment-naïve at commencement of miglustat and 25 (64\%) had previously received ERT for $\geq 5$ years. The median previous ERT dose among switch patients was $30 \mathrm{U} / \mathrm{kg}$ once every other week: nine were receiving ERT at $>30 \mathrm{U} / \mathrm{kg}$ every other week at switching.

Among all patients who currently remain on miglustat, the mean (range) overall duration of miglustat treatment is 89.2 (6-120) months. Thirty-one patients (79\%) have received miglustat for $\geq 5$ years, and 15 (38\%) have been on miglustat for 12 years. All currently treated patients have undergone at least one post-baseline follow-up visit, providing a median (range) efficacy follow-up period of 99 (9-120) months.

\subsection{Core disease parameters}

Absolute values of $\mathrm{Hb}$ concentration, platelet count, spleen and liver volume, and plasma CHT and CCL18/PARC levels in the entire cohort $(N=63)$ at baseline and annual follow-up are summarized in Fig. 1. Haemoglobin concentration increased between baseline and Year 4, and appeared stable thereafter (Fig. 1a). Overall, there were no statistically significant differences in mean $\mathrm{Hb}$ versus baseline at 2, 5 or 10 years of follow up. Among patients with available data and anaemia at baseline $(n=6)$, the mean (SD) change in Hb concentration versus baseline was $+1.3 \mathrm{~g} / \mathrm{dL}(0.74)$ at 6 -month follow up, and $\mathrm{Hb}$ remained stable thereafter. In switch patients with available data $(n=3)$, Hb concentration was $<12 \mathrm{~g} / \mathrm{dL}$ at the time of switching and improved by $+1.8 \mathrm{~g} / \mathrm{dL}(0.39)$ versus baseline at subsequent follow up.

Platelet counts appeared stable over the 12-year course of follow up, and there was no statistically significant overall change from baseline in mean platelet counts at 2-, 5- or 10-year follow-up (Fig. 1b). Among 20 patients with available data at 10-year follow up, absolute values had decreased slightly, with a mean (range) change from baseline of -35 $(10-86) \times 10^{9} / \mathrm{L}$. Four patients with available data had a platelet count $<100 \times 10^{9} / \mathrm{L}\left(70,77,90\right.$ and $\left.95 \times 10^{9} / \mathrm{L}\right)$ at final follow up.

Table 1

Patient and disease characteristics at baseline (all miglustat-treated patients; $N=63$ ).

\begin{tabular}{lll}
\hline Characteristic & $\begin{array}{l}\text { Treatment-naïve } \\
(N=20)\end{array}$ & $\begin{array}{l}\text { Switch } \\
(N=43)\end{array}$ \\
\hline $\begin{array}{l}\text { Age in years, median (range) } \\
\text { Male: female, n (\%) }\end{array}$ & $\begin{array}{l}55.7(18-79) \\
11(55.0): 9(45.0)\end{array}$ & $\begin{array}{l}48.4(21-70) \\
23(53.5): 20(46.5)\end{array}$ \\
Genotype, n (\%): & & \\
N370S/N370S & $6(30.0)$ & $3(6.9)$ \\
N370S/L444P & $6(30.2)$ & $13(30.2)$ \\
N370S/other & $8(40.0)$ & $19(44.1)$ \\
Other/other & 0 & $8(18.6)$ \\
SSI, median (range) & $6.6(3.0-7.5)$ & $6.6(2.8-7.6)$ \\
Time on ERT (years) & 0 & $6.9(1.2-13.5)$ \\
Splenectomy (\%) & $3(15.0)$ & $7(16.3)$ \\
\hline
\end{tabular}

ERT, enzyme replacement therapy; SSI, severity score index.
Spleen and liver volumes did not show statistically significant variations over the 10-year course of follow-up (Figs. 1c and d).

\subsection{Biomarkers}

At 10-year follow up, we observed wide variability in CHT activity with increased mean in $24 / 42(57 \%)$ patients with available data $(+2448 \mathrm{nmol} / \mathrm{mL} \cdot \mathrm{h}$; range $135-13,687)$, and CCL18/PARC was also increased in 18/42 (43\%) patients (mean $250 \mathrm{ng} / \mathrm{mL}$; range 19-1016). These increases were not statistically significant versus baseline values ( $p=0.41$ and $p=0.08$, respectively) and were not associated with clinical manifestations (Figs. 1e and f).

\subsection{Therapeutic goals analysis}

Over $80 \%$ of patients achieved or maintained accepted therapeutic goals for GD1 in the switched on miglustat therapy during the study observation period. Haemoglobin concentration was normalised (to $>11.0 \mathrm{~g} / \mathrm{dL}$ for women; $>12.0 \mathrm{~g} / \mathrm{dL}$ for men) after $12-24$ months on miglustat, independent of transfusions. No symptoms of anaemic syndrome were identified over the initial 2 years of follow up. Overall, $\mathrm{Hb}$ values were stable at 2-year follow up, and remained so up to the end of the 10-year observation period (Fig. 1) Platelet counts increased in treatment-naïve patients with moderate thrombocytopenia $\left(40-99 \times 10^{9} / \mathrm{L}\right)$ during the first 12 months of miglustat therapy, and continued improvement up to 60 months. Among switch patients, platelet counts were maintained at $>100 \times 10^{9} / \mathrm{L}$ throughout 10 -year follow-up. The accepted goal for reducing spleen volume is a $30-50 \%$ decrease in the first year and $50-60 \%$ at 5 years, and the target reduction in liver volume is $20-30 \%$ over $12-24$ months and $30-40 \%$ after 3-5 years. Both of these goals were achieved during miglustat therapy in this cohort.

\subsection{Bone parameters}

The majority of patients with available bone data (32/43 [74.4\%]) reported an overall reduction in chronic bone pain. Mean (range) overall S-MRI scores at baseline and 2-year follow up were 9.6 points $(0-25)$ and 7.2 points $(0-21)$, respectively $(p=0.042)$. The mean S-MRI score in the treatment-naïve group was 8.8 points (0-14) and 10.0 $(0-25)$ in the switch group at start therapy. Ultrasound examinations of calcaneus bone among a total of 24 patients who received miglustat for $\geq 2$ years (seven treatment-naïve and 17 switch patients) indicated bilateral statistically significant increases in BMD (Table 2).

\subsection{Tolerability and safety}

The most commonly recorded adverse events in the whole study cohort $(N=63)$ were mild-to-moderate gastrointestinal disturbances, recorded in 25 (40\%) patients. Gastrointestinal adverse events were reversible and were improved by adherence to a controlled diet or disaccharidase supplementation in most cases. An exploratory comparative subgroup analysis to identify differences in general characteristics between patients who showed good gastrointestinal tolerability $(n=38)$ and those in whom miglustat was not well tolerated $(n=25)$ did not reveal any significant differences (data not shown). Gastrointestinal disturbances were recorded as the reason for miglustat discontinuation in a total of 11 patients.

Thirty-eight patients (60\%) experienced a reversible fine-hand tremor without functional consequences during the first month of therapy, but only 20 patients reported persistence of this effect after 4 years on therapy. The tremor was reversible upon discontinuation of therapy. Electrophysiological signs of subclinical peripheral neuropathy were observed in $8 \%$ of patients before miglustat initiation. Periodic neurological tests and electromyography (EMG) in these patients during follow- 


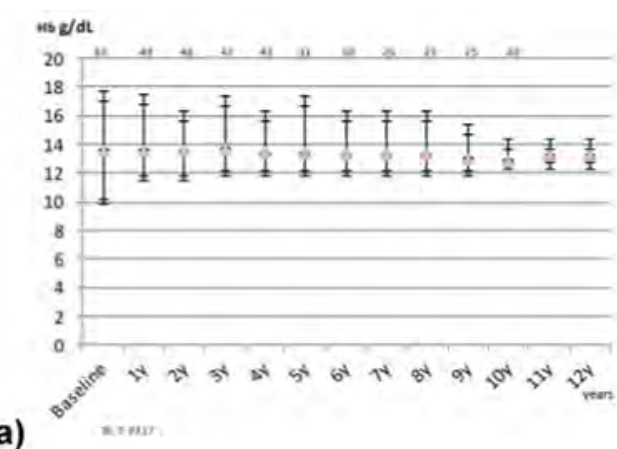

c)

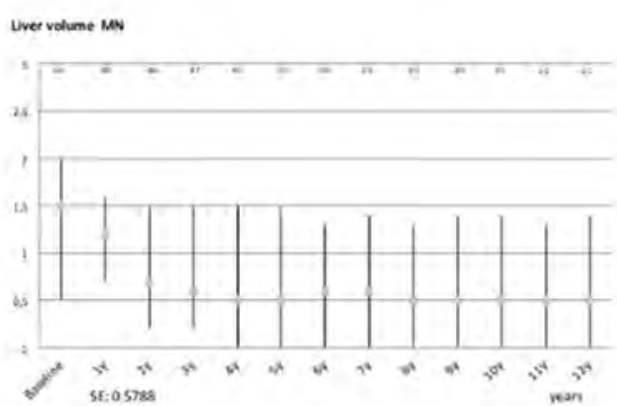

e)

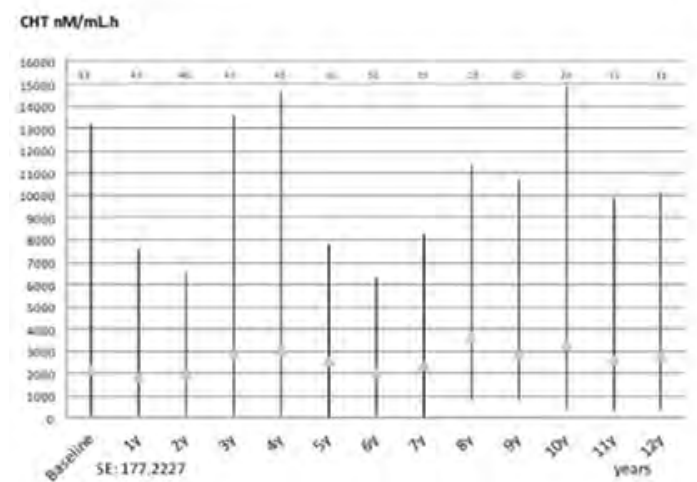

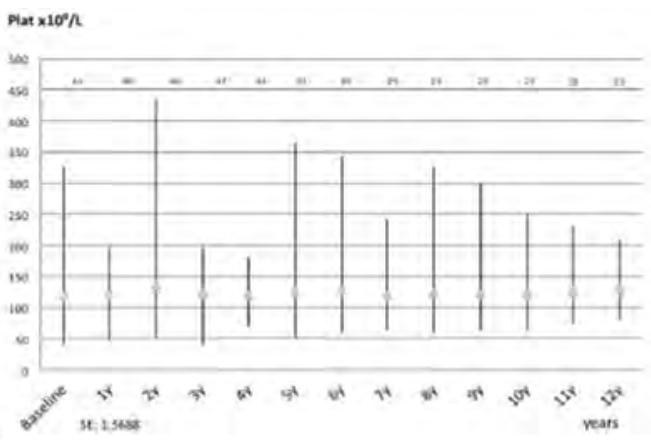

d)

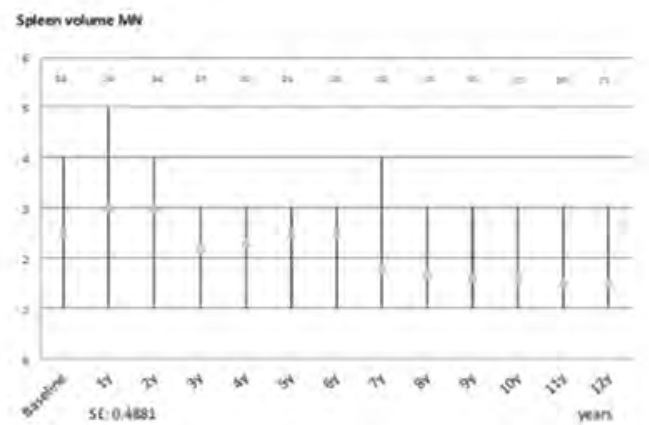

f)

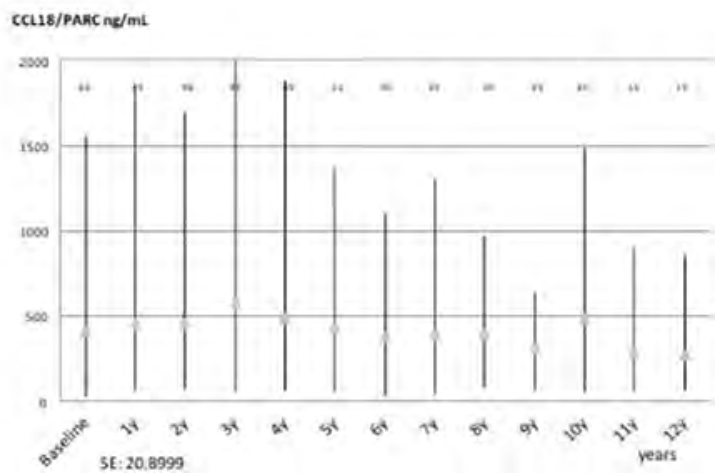

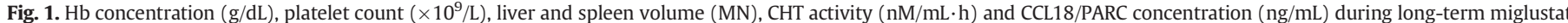

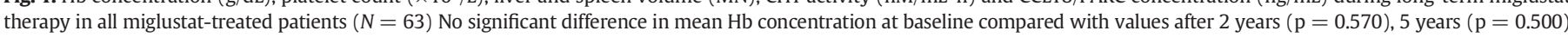

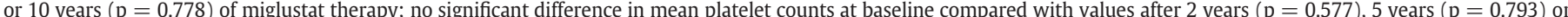

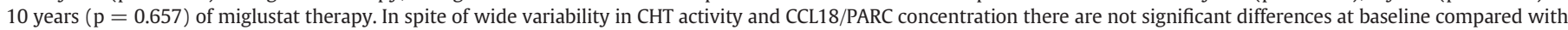
values after 2, 5 and 10 years (CHT Baseline vs 10 years $(p=0.41))$, (CCL18/PARC Baseline vs 10 years $(p=0.08))$.

Table 2

Broadband ultrasound attenuation values, and derived Z-score and T-score in calcaneus bone assessments in 24 patients ${ }^{\mathrm{b}}$ treated with miglustat for 2 years.

\begin{tabular}{|c|c|c|c|c|c|c|c|c|}
\hline \multirow[t]{2}{*}{ Limb } & \multicolumn{2}{|c|}{$\mathrm{BUA}^{\mathrm{a}}$, mean (range) } & \multirow[t]{2}{*}{$\mathrm{P}^{\mathrm{c}}$} & \multirow[t]{2}{*}{ Score } & \multicolumn{2}{|c|}{ Z-T score, mean (SD) } & \multirow{2}{*}{$\begin{array}{l}\text { Change } \\
95 \% \mathrm{CI}\end{array}$} & \multirow[t]{2}{*}{$\mathrm{P}^{\mathrm{c}}$} \\
\hline & Baseline & Month 24 & & & Baseline & Month 24 & & \\
\hline \multirow[t]{2}{*}{ Right } & $82.4(47-101)$ & $84.2(66-100)$ & 0.04 & Z & $-1.3(0.9)$ & $-1.1(0.9)$ & $0.09,0.42$ & $<0.01$ \\
\hline & & & & $\mathrm{T}$ & $-1.3(0.9)$ & $-1.3(0.9)$ & $0.09,0.48$ & $<0.01$ \\
\hline \multirow[t]{2}{*}{ Left } & $74.2(32-100)$ & $75.6(48-101)$ & 0.06 & $\mathrm{Z}$ & $-1.1(0.9)$ & $-0.9(0.5)$ & $0.09,0.30$ & $<0.01$ \\
\hline & & & & $\mathrm{T}$ & $-1.5(1.1)$ & $-1.1(0.4)$ & $0.08,0.45$ & $<0.01$ \\
\hline
\end{tabular}

a Broadband ultrasound attenuation.

b Data based on seven treatment-naïve patients and 17 previous ERT patients with available data.

c P-values related to a comparison of month 24 values with baseline values, as assessed based on the paired $t$-test. 
up did not reveal any evidence of progressive neuropathy. However during follow-up two patients developed a peripheral neuropathy.

No patients in the study appeared to develop cognitive deterioration during miglustat therapy for up to 12 years. Weight loss $>10 \%$ was reported for three patients (4.7\%). Among patients who reported gastrointestinal disturbances as the reason for miglustat discontinuation, six also showed increased bone infiltration, two had a bone crisis, and one had $>10 \%$ weight loss. One patient also had Gaucher cell infiltration in the myocardium (at biopsy), and one had persistent headache that led to discontinuation in the early stages of treatment.

Kaplan-Meier estimate of the probability of drug discontinuation or death in the entire cohort $(n=63)$ indicated a tendency to lower overall rate of miglustat discontinuation among treatment-naïve patients compared with the switch group, but the comparison by Log-Rank test is not significant. (Fig. 2).

\section{Discussion}

We present long-term data on changes in haematological and visceral indices and biomarkers in both treatment-naïve and ERTstabilized adult GD1 patients treated with miglustat for up to 12 years in clinical practice settings. To our knowledge, this report represents the longest real-world assessment of the effectiveness and safety of miglustat in GD1 to date, and builds on previous findings from this cohort and on data reported in other open-label studies and case series $[15,17,18,19,23,24]$.

Throughout up to 12 years of therapy with miglustat in this Spanish cohort, $\mathrm{Hb}$ concentration remained largely unchanged and platelet count remained stable, with moderate fluctuations. Liver and spleen volumes were also stable during follow up. These findings are consistent with accepted therapeutic goals for GD1 therapy in treatment-naïve patients reported by Pastores et al. in 2004 [6,7]. The data from this cohort add further support for the use of miglustat in patients who refuse ERT or who are otherwise unsuited to regular intravenous infusions. The efficacy of miglustat as a maintenance monotherapy for GD1 over 24 months has previously been reported in 36 patients previously stabilized with ERT [25]. In the observational, retrospective multicentre study in 115 miglustat-treated patients reported by Kuter et al. [19], $\mathrm{Hb}$ and platelet counts tended to increase in treatment-naïve patients during miglustat therapy, and remained stable or decreased slightly in ERT pre-treated patients.

The efficacy of miglustat may result from two mechanisms of action. Besides inhibiting substrate formation, miglustat may also accelerate

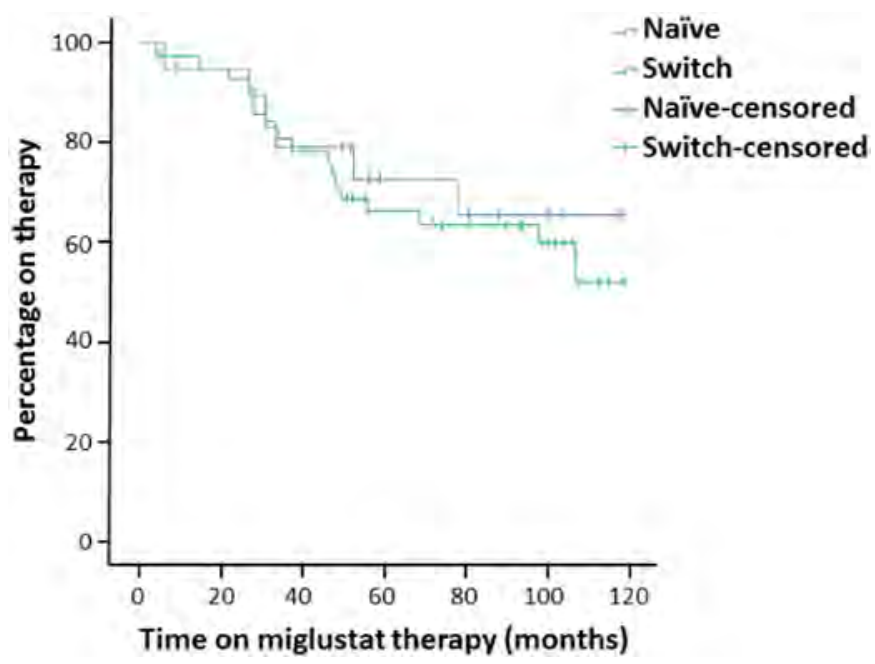

Fig. 2. Estimated probability to discontinuation of miglustat therapy (all miglustat-treated patients; $N=63$ ) Data censored to account for discontinuation of miglustat therapy or death from any cause (Log-Rank test 0.482 ). destruction of the glycolipid complex by increasing glucocerebrosidase (GC) activity through a chaperoning function. The chaperoning effect of miglustat was shown to be specific for glucocerebrosidase with mutations in the catalytic domain (domain III) $[25,26]$. In vitro studies demonstrated that glucocerebrosidase activity was increased 2.5-fold in cells transfected with the N370S mutation when cultured in a medium containing miglustat [26]. It is therefore noteworthy that the majority of the patients in our series had at least one glucocerebrosidase gene allele with the N370S mutation.

Disease biomarkers during miglustat therapy showed a tendency to increase during follow up in this study without any appearance of clinical manifestations. It is therefore possible that differences in patient compliance or inter-individual variations affected the observed CHT values. Overall our data suggest that plasma CHT and CCL18/PARC might not fully reflect systemic disease burden in GD1, and cast doubt on the strict validity of these biomarkers in clinical decision making [25].

Bone disease is the most frequent and debilitating manifestation of GD1 [27]. A prospective pooled analysis of data from three open-label studies with miglustat as monotherapy for GD1 demonstrated that miglustat reduces the incidence of bone pain and improves BMD [28] - an effect thought to stem from the fact that this agent has a wide distribution throughout body tissues, including bone [29]. Early and sustained increases in lumbar spine and femoral neck BMD were reported, with significant increases from baseline evident at 6,12 and 24 months [28]. For these reasons, miglustat might be a valuable treatment option for the improvement of bone disease in GD1.

Based on the current cohort we assessed long-term changes in BMD and bone elasticity using ultrasound methodology in the calcaneus (heel) bone. Data from heel bone ultrasound analyses have been shown to correlate well with DXA measurements, although ultrasound data indicate greater degrees of change over time compared with DXA findings [30]. Evaluations in our patients were performed by the same radiologist using the same machine, and according to the same protocol, at baseline and follow up, and showed statistically significant increases of both ultrasound-derived BUA and DXA-derived Z- and T-scores after 2 years on miglustat. The majority of patients in the whole cohort also showed improvements in chronic bone pain and Gaucher cell infiltration of bone (as measured by S-MRI) during 2 years on therapy.

Avascular necrosis was observed in two patients (3.1\%), both of whom were non-splenectomized switch patients, and one of which did not demonstrate good adherence to miglustat therapy. GD1 is a chronic disorder and requires an effective and continuous treatment regimen on a life-long basis. For patients on prolonged oral therapy the correct adherence to every day therapy is a widespread problem [31].

Gastrointestinal tolerability showed a high degree of individual variability in this study, which was possibly related to differences in food habits and levels of disaccharidase inhibition (Medrano B et al. in press). Similar to therapies for other chronic diseases such as diabetes, arterial hypertension and dyslipidaemia, gastrointestinal disturbances such as diarrhoea, flatulence and vomiting are known, common effects seen during miglustat treatment [12,13]: it has been estimated that approximately $56 \%$ of drug therapies have an influence on gastrointestinal function. Although uncomfortable if they occur, gastrointestinal adverse events related to miglustat therapy are reversible and do not require intensive therapy in most cases [31]. While generally moderate in intensity and not related to severe complications, gastrointestinal effects were the most frequent reason for miglustat discontinuation in this cohort, which is in line with previous published data [31].

Tremor was the main neurological side effect observed during the study, particularly at commencement of treatment. Two new cases of peripheral neuropathy with clinical symptoms that were attributable to miglustat were observed in this study. Previous published data demonstrating that peripheral neuropathy should be considered part of the natural history of GD1 [32,33,34], and data from up to 9 years of postmarketing clinical safety surveillance for miglustat that showed no 
association between miglustat and peripheral neuropathy [17,23], however treating physicians should be aware of this association to improve early detection.

No other adverse long-term effects of miglustat treatment have been documented [17,23].

\section{Conclusions}

The first trials of oral SRT with miglustat in GD1 began $>16$ years ago $[14,25,35,36]$. As a result there is now considerable, widespread experience of its use in clinical practice. The general opinion regarding oral miglustat therapy is that it is satisfactory both in terms of controlling disease in treatment-naïve patients and in maintaining disease stability in patients previously stabilized on ERT. Miglustat is not a curative therapy, but it does prevent excess lipid storage in the spleen, liver and bone marrow, and has been shown to maintain disease stability in line with established therapeutic goals in the majority of patients. Overall, longterm treatment with miglustat appeared associated with only mild-tomoderate adverse events in this Spanish cohort. Importantly, miglustat also provided additional benefits on bone parameters, with statistically significant decreases in bone marrow infiltration (as measured by SMRI) and increases in BMD (as measured by BUA and DXA). Plasma CHT activity and CCL-18/PARC concentration showed a trend to increase over time, but did not reach initial values. As an aside, biomarker alterations did not correlate with clinical.

As we have commented in previous reports $[15,16]$, and reinforce now with the benefit of accumulated experience, miglustat appears to be a valid alternative to ERT as a maintenance therapy in GD1. This agent also has the added advantage of convenient oral administration, which serves as a useful therapeutic option for maintaining efficacy in patients who discontinue ERT due to preference, holidays, travel, work or other commitments.

\section{Funding sources}

This work was partially sponsored by a grant from FEETEG (01-04), FIS: EC07/90737, 07/90938, PS09/02556, and PS12/01219. PG has received research funding and consultancy fees from Actelion Pharmaceuticals Ltd. All authors are members of the Spanish Study Group on Gaucher Disease, which is supported by the Spanish Gaucher Disease Foundation (FEETEG).

\section{Author contributions}

PG is principal investigator for the Spanish Registry of Gaucher Disease and coordinated the study, analysed and interpreted the data, and drafted the manuscript; PA contributed to the enzymatic, genetic and serum biomarker investigation, and to the statistical analyses; PL contributed to the statistical analysis and to the manuscript; MP helped to conduct the study, interpreted the data, and provided review input on the manuscript. Other authors collected clinical, analytical and follow-up clinical data, contributed to data analyses. All authors revised and approved the final draft for submission.

\section{Acknowledgements}

Matthew Reilly PhD at InTouch Medical Ltd. provided medical editing support in the preparation of this manuscript for submission, paid for by Actelion Pharmaceuticals.

\section{References}

[1] E. Beutler, G.A. Grabowski, Gaucher disease, in: C.R. Scriver, A.L. Beaudet, W.S. Sly, D. Valle (Eds.), The Metabolic and Molecular Basis of Inherited Disease, McGraw-Hill, New York 1995, pp. 3635-3668.
[2] T.M. Cox, J.P. Schofield, Gaucher's disease: clinical features and natural history, Baillieres Clin. Haematol. 10 (1997) 657-689.

[3] N.W. Barton, R.O. Brady, J.M. Dambrosia, et al., Replacement therapy for inherited enzyme deficiency-macrophage-targeted glucocerebrosidase for Gaucher's disease, N. Engl. J. Med. 324 (1991) 1464-1470.

[4] A. Zimran, D. Elstein, R. Kannai, et al., Low-dose enzyme replacement therapy for Gaucher's disease: effects of age, sex, genotype, and clinical features on response to treatment, Am. J. Med. 97 (1994) 3-13.

[5] K. Starzyk, S. Richards, J. Yee, S.E. Smith, W. Kingma, The long-term international safety experience of imiglucerase therapy for Gaucher disease, Mol. Genet. Metab. 90 (2007) 157-163.

[6] G.M. Pastores, P. Giraldo, P. Cherin, A. Mehta, Goal-oriented therapy with miglustat in Gaucher disease, Curr. Med. Res. Opin. 25 (2009) 23-37.

[7] G.M. Pastores, N.J. Weinreb, H. Aerts, et al., Therapeutic goals in the treatment of Gaucher disease, Semin. Hematol. 41 (2004) 4-14.

[8] D.I. Rosenthal, S.H. Doppelt, H.J. Mankin, et al., Enzyme replacement therapy for Gaucher disease: skeletal responses to macrophage-targeted glucocerebrosidase Pediatrics 96 (1995) 629-637.

[9] D. Elstein, I. Hadas-Halpern, M. Itzchaki, et al., Effect of low-dose enzyme replacement therapy on bones in Gaucher disease patients with severe skeletal involvement, Blood Cells Mol. Dis. 22 (1996) 104-111 (discussion 112-4).

[10] E. Beutler, A. Demina, K. Laubscher, et al., The clinical course of treated and untreated Gaucher disease. A study of 45 patients, Blood Cells Mol. Dis. 21 (1995) 86-108.

[11] P. Actelion, 2010. in: Zavesca ${ }^{\circledR}$ (miglustat capsules) (Ed.), Prescribing Information, 2010 Available at, http://www.zavesca.com/pdfs/zavesca_pi.pdf (Accessed 9 March 2010).

[12] Actelion, Zavesca ${ }^{\circledR}$ (miglustat capsules), Prescribing Information, Available at, 2010 http://www.zavesca.com/pdfs/zavesca_pi.pdf (Accessed 9 June 2011).

[13] Actelion, Miglustat (Zavesca), Summary of Product Characteristics, EMA (EudraPharm), 2014.

[14] T. Cox, R. Lachmann, C. Hollak, et al., Novel oral treatment of Gaucher's disease with N-butyldeoxynojirimycin (OGT 918) to decrease substrate biosynthesis, Lancet 355 (2000) 1481-1485.

[15] P. Giraldo, P. Alfonso, K. Atutxa, et al., Real-world clinical experience with long-term miglustat maintenance therapy in type 1 Gaucher disease: the ZAGAL project, Haematologica 94 (2009) 1771-1775.

[16] P. Giraldo, P. Latre, P. Alfonso, et al., Short-term effect of miglustat in every day clinical use in treatment-naive or previously treated patients with type 1 Gaucher's disease, Haematologica 91 (2006) 703-706.

[17] C.E. Hollak, D. Hughes, I.N. van Schaik, B. Schwierin, B. Bembi, Miglustat (Zavesca) in type 1 Gaucher disease: 5-year results of a post-authorisation safety surveillance programme, Pharmacoepidemiol. Drug Saf. 18 (2009) 770-777.

[18] T.M. Cox, D. Amato, C.E. Hollak, et al., Evaluation of miglustat as maintenance therapy after enzyme therapy in adults with stable type 1 Gaucher disease: a prospective, open-label non-inferiority study, Orphanet J. Rare Dis. 7 (2012) 102.

[19] D.J. Kuter, A. Mehta, C.E. Hollak, et al., Miglustat therapy in type 1 Gaucher disease: clinical and safety outcomes in a multicenter retrospective cohort study, Blood Cells Mol. Dis. 51 (2013) 116-124.

[20] T.M. Cox, J.M. Aerts, G. Andria, et al., The role of the iminosugar Nbutyldeoxynojirimycin (miglustat) in the management of type I (non-neuronopathic) Gaucher disease: a position statement, J. Inherit. Metab. Dis. 26 (2003) 513-526.

[21] M. Roca, J. Mota, P. Alfonso, M. Pocovi, P. Giraldo, S-MRI score: a simple method for assessing bone marrow involvement in Gaucher disease, Eur. J. Radiol. 62 (2007) 132-137.

[22] http://feeteg.org/G_conocer.php. Accessed 30 September 2016 (2016)

[23] M. Brand, A. Muller, J. Alsop, et al., Results from a 9-year Intensive Safety Surveillance Scheme $(\mathrm{IS}(3))$ in miglustat (Zavesca((R)))-treated patients, Pharmacoepidemiol. Drug Saf. 24 (2015) 329-333.

[24] C. Serratrice, L. Swiader, J. Serratrice, Switching from imiglucerase to miglustat for the treatment of French patients with Gaucher disease type 1: a case series, J. Med. Case Rep. 9 (2015) 146.

[25] D. Elstein, A. Dweck, D. Attias, et al., Oral maintenance clinical trial with miglustat for type I Gaucher disease: switch from or combination with intravenous enzyme replacement, Blood 110 (2007) 2296-2301.

[26] P. Alfonso, S. Pampin, J. Estrada, et al., Miglustat (NB-DNJ) works as a chaperone for mutated acid beta-glucosidase in cells transfected with several Gaucher disease mutations, Blood Cells Mol. Dis. 35 (2005) 268-276.

[27] B. Brumshtein, H.M. Greenblatt, T.D. Butters, et al., Crystal structures of complexes of $\mathrm{N}$-butyl- and $\mathrm{N}$-nonyl-deoxynojirimycin bound to acid beta-glucosidase: insights into the mechanism of chemical chaperone action in Gaucher disease, J. Biol. Chem. 282 (2007) 29052-29058.

[28] P.B. Deegan, E. Pavlova, J. Tindall, et al., Osseous manifestations of adult Gaucher disease in the era of enzyme replacement therapy, Medicine (Baltimore) 90 (2011) $52-60$.

[29] G.M. Pastores, D. Elstein, M. Hrebicek, A. Zimran, Effect of miglustat on bone disease in adults with type 1 Gaucher disease: a pooled analysis of three multinational, open-label studies, Clin. Ther. 29 (2007) 1645-1654.

[30] A. Treiber, O. Morand, M. Clozel, The pharmacokinetics and tissue distribution of the glucosylceramide synthase inhibitor miglustat in the rat, Xenobiotica 37 (2007) 298-314

[31] M.I. El-Desouki, M.S. Sherafzal, S.A. Othman, Comparison of bone mineral density with dual energy X-ray absorptiometry, quantitative ultrasound and single energy X-ray absorptiometry, Saudi Med. J. 26 (2005) 1346-1350.

[32] N. Belmatoug, A. Burlina, P. Giraldo, et al., Gastrointestinal disturbances and their management in miglustat-treated patients, J. Inherit. Metab. Dis. 34 (2011) 991-1001. 
[33] M. Biegstraaten, E. Mengel, L. Marodi, et al., Peripheral neuropathy in adult type 1 Gaucher disease: a 2-year prospective observational study, Brain 133 (2010) 2909-2919.

[34] J.L. Capablo, A. Saenz de Cabezon, J. Fraile, et al., Neurological evaluation of patients with Gaucher disease diagnosed as type 1, J. Neurol. Neurosurg. Psychiatry 79 (2008) 219-222.
[35] D. Elstein, C. Hollak, J.M. Aerts, et al., Sustained therapeutic effects of oral miglustat (Zavesca, N-butyldeoxynojirimycin, OGT 918) in type I Gaucher disease, J. Inherit. Metab. Dis. 27 (2004) 757-766.

[36] G.M. Pastores, N.L. Barnett, E.H. Kolodny, An open-label, noncomparative study of miglustat in type I Gaucher disease: efficacy and tolerability over 24 months of treatment, Clin. Ther. 27 (2005) 1215-1227. 